\title{
THE 1991 MARCH 22 FLARE: POSSIBLE ANISOTROPY OF HIGH-ENERGY NEUTRAL EMISSION
}

\author{
L. G. KOCHAROV, ${ }^{*}$ JEONGWOO W. LEE, H. WANG and H. ZIRIN \\ Big Bear Solar Observatory, California Institute of Technology, Pasadena, CA 91125, U.S.A. \\ and \\ G. A. KOVALTSOV and I. G. USOSKIN \\ A. F. Ioffe Physical-Institute, St. Petersburg, 194021, Russia
}

(Received 17 August, 1994; in revised form 3 January, 1995)

\begin{abstract}
We made a parameter fit to the Haleakala neutron monitor counting rate during the 1991 March 22 solar flare (Pyle and Simpson, 1991) using the time profiles of $\gamma$-rays at $0.42-80 \mathrm{MeV}$ obtained with the GRANAT satellite (Vilmer et al., 1994) and the microwave data from Owens Valley Radio Observatory. We use a two-component neutron injection function to find that either an impulsive injection or the 'impulsive-plus-prolonged' neutron injection is possible. In both cases, the number of $>300 \mathrm{MeV}$ neutrons emitted towards the Earth is estimated as $\approx 2 \times 10^{27} \mathrm{sr}^{-1}$, which is less than that of the 1990 May 24 flare by an order of magnitude.

We tested if such a big difference in neutron number detected on the Earth can be accounted for solely by their different positions on the solar disk. For the estimation of the degree of anisotropy of high-energy secondary emission, we made use of macroscopic parameters of the flare active region, in particular, the vector magnetogram data from the Big Bear Solar Observatory. In our result, the anisotropy factor for the neutral emissions of the 1991 March 22 flare is only $\approx 1-10$, which is rather small compared with previous theoretical predictions for a disk flare. Such a moderate anisotropy is due to the relatively large inclination angles of the magnetic fields at the footpoints of the flaring loop where accelerated particles are trapped. We thus concluded that the smaller number of neutrons of the 1991 March 22 flare would be not only due to its location on the disk, but also due to fewer protons accelerated during this event as compared with the 1990 May 24 limb event. For a more precise determination of the anisotropy factor in a flare, we need a detailed spectrum of electron bremsstrahlung in $0.1-10 \mathrm{MeV}$ and the fluence of $\gamma$-ray emission from the $\pi^{0}$-decay.
\end{abstract}

\section{Introduction}

The 1991 March 22 flare is one of the biggest flares that occurred in the last solar maximum. It attained GOES soft X-ray class X9.4. During this event an increase of counting rate was recorded in the Haleakala neutron monitor (NM) (Pyle and Simpson, 1991) and the $\gamma$-ray bursts were detected by the GRANAT satellite (Vilmer et al., 1994; Pelaez, 1993). Meanwhile, a multifrequency observation of the microwave bursts in the range of $1-18 \mathrm{GHz}$ was made by the Solar Array at the Owens Valley Radio Observatory (OVRO) to uncover a characteristic spectral activity indicative of a dynamic evolution of the source (Lee et al., 1994). $\mathrm{H} \alpha$ and the vector magnetogram observation of the flaring region were made at the Big Bear Solar Observatory (BBSO) allowing the information of the source morphology including the inclination angles of magnetic fields. Such a data set provides an

* Visting Associate from St. Petersburg State Technical University, St. Petersburg, 195251, Russia.

Solar Physics 158: 95-114, 1995.

(C) 1995 Kluwer Academic Publishers. Printed in Belgium. 
opportunity to explore the production of energetic particles and their interaction with ambient matter from which the secondary radiations are emitted.

Recently, we have performed a correlative study of the 1990 May 24 flare in which the Climax NM counting rates and the $2.2 \mathrm{MeV} \gamma$-ray line and the $\gamma$-ray continuum are analyzed and the results are interpreted based on the microwave and optical observations (Kocharov et al., 1994). It was shown that the secondary emission observed on 1990 May 24 may be explained as originating from the two sources (magnetic loops). The smaller source with a scale of $\approx 2 \times 10^{9} \mathrm{~cm}$ gives rise to the fast-decaying component of high-energy emission, while the larger source with a scale of $\approx 2 \times 10^{10} \mathrm{~cm}$ is responsible for the microwave radiation and the slowly-decaying neutron in jection. Now we apply the similar technique to the 1991 March 22 solar flare. It appears that the 1991 March 22 flare shares several common properties with the 1990 May 24 one in terms of microwave flux spectra and timing and strength of shock waves as well as soft X-ray fluxes. First, according to the OVRO data, both flares produced huge microwave flux amounting to $\approx 10^{4}$ s.f.u. which persists from 1 to $18 \mathrm{GHz}$. Second, both events showed a spectral shift of dominant flux from high frequencies at the initial rise to low frequencies at the decay phase (Lee et al., 1994). It is also noteworthy that $\mathrm{H} \alpha$ movies obtained at BBSO reveal the presence of Moreton waves in both flares and that in both events the timing of shock waves relative to the peak time of microwaves and $\gamma$-rays suggests that the shock wave may be responsible for the impulsive acceleration of electrons and protons. Thus, information gathered through the microwave and $\mathrm{H} \alpha$ observations points to a possibility that the acceleration of particles and their subsequent evolution during both flares may have taken place under a similar source condition.

On the other hand, these two flares show a large difference in their total numbers of high-energy neutrons injected towards the Earth. As we shall show in Section 3, this number for the 1991 March 22 flare is estimated as $N_{n}(>300 \mathrm{MeV}) \approx$ $2 \times 10^{27} \mathrm{sr}^{-1}$, which is less than that for the 1990 May 24 flare by an order of magnitude. Noting that the 1990 May 24 flare was a limb event while this 1991 March 22 flare occurred at an angular distance of only $\approx 39^{\circ}$ from the disk center, such a difference in the neutron number detected on Earth is, in fact, expected if there is anisotropy in neutron generation at the Sun. There have been many discussions as to the anisotropy of high-energy neutral (i.e., neutron and $\gamma$ ray) emissions from both the theoretical and the empirical viewpoints (Petrosian, 1985; Bai, 1988; Vestrand et al., 1987, 1991; Miller and Ramaty, 1989; Kocharov and Kovaltsov, 1990, and references therein). However, the precise value of the anisotropy is yet uncertain. It thus becomes interesting whether the difference of the neutron number observed in the 1991 March 22 flare from that in the 1990 May 24 flare was solely due to their different locations on the solar disk.

In this paper, we study the neutron injection during the 1991 March 22 flare as a function of time and energy by analyzing the neutron counting rates from the Haleakala NM and the $\gamma$-ray intensities from the GRANAT satellite. We thus 
consider several possible scenarios for the neutron injection, taking into account the degree of the anisotropy of neutron emission in each scenario as the most important check-point for its validity. For the estimation of possible anisotropy of the neutron emission, we make use of the detailed morphology of magnetic fields as learned from the vector magnetogram data of BBSO. The plan of this paper is as follows: in Section 2, we report the optical and the microwave observations. In Section 3, we describe the results of the Haleakala NM counting rates and the $\gamma$-ray observation by the GRANAT/PHEBUS and make a parameter fit to the NM data. In Section 4, we briefly review the theoretical predictions for the anisotropy of high-energy secondary emission and apply it to the present results for their interpretation. Based on these results, we discuss the nature of the 1991 March 22 flare in Section 5. Finally, a summary of our work will be given in Section 6 .

\section{Optical and Microwave Emissions}

The X9.4/3B solar flare on 1991 March 22 occurred at 22:41-23:17 UT in the active region NOAA 6555 located at S26 E28. Figure 1 shows three frames of $\mathrm{H} \alpha$ obtained at BBSO showing three different stages of $\mathrm{H} \alpha$ flare development. It is seen that the $\mathrm{H} \alpha$ brightening began from a low-lying small loop (Figure 1(a)); it rapidly expanded to touch a remote region (Figure 1(b)); and a big cloud of $\mathrm{H} \alpha$ brightening was formed (Figure 1(c)). As seen in the first frame, the projected length of the small loop where the $\mathrm{H} \alpha$ kernel occurred is $\approx 30^{\prime \prime}\left(\approx 2 \times 10^{9} \mathrm{~cm}\right)$. In the last frame, the $\mathrm{H} \alpha$ brightening expanded to its maximum size which amounted to $\approx 100^{\prime \prime} \times 100^{\prime \prime}$, and it lasted over $25 \mathrm{~min}$, gradually diminishing afterwards.

A set of vector magnetograms was also obtained at BBSO over this active region from 21:40 UT until 23:50 UT. As a result, both longitudinal and transverse magnetic fields were measured. The configuration of the device used to measure the transverse magnetic fields is described by Cacciani, Varsik, and Zirin (1989). In Figure 2(a), we show a vector magnetogram in which the longitudinal $\left(B_{\|}\right)$ and the transverse magnetic fields $\left(B_{\perp}\right)$ are represented by contours and bars, respectively. We use these measurements to get the inclination angle of magnetic fields relative to the line of sight as $\theta \equiv \tan ^{-1}\left[B_{\perp} / B_{\|}\right]$. In Figure 2(b), we plot the distribution of the angles in a gray scale where the angle varies from $\theta=0^{\circ}$ at the dark region to $\theta=90^{\theta}$ at the white region. Also specified with the brackets in Figure 2(b) is the place of He D3 brightening, which is believed to be due to heating by energetic particles precipitating into the lower chromosphere (Zirin, 1988). We note that the magnetic inclination angles in this region lie mostly in the range of $45 \leq \theta<90^{\circ}$.

The Moreton wave was seen during the 1991 March 22 flare as a propagating disturbance in $\mathrm{H} \alpha$ images. Using full-disk $\mathrm{H} \alpha$ images obtained at $\mathrm{BBSO}$ we were able to locate the passage of the shock wave by noting the activation of $\mathrm{H} \alpha$ filaments as the shock waves passed by. As a result, the mean speed of the shock 
(a)

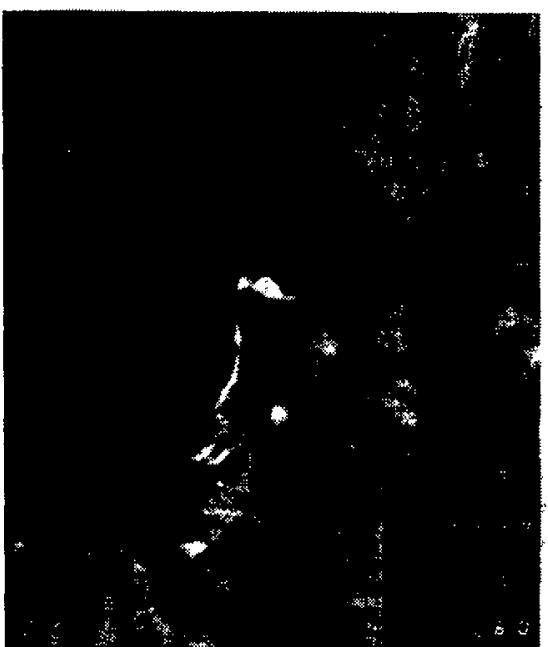

(b)

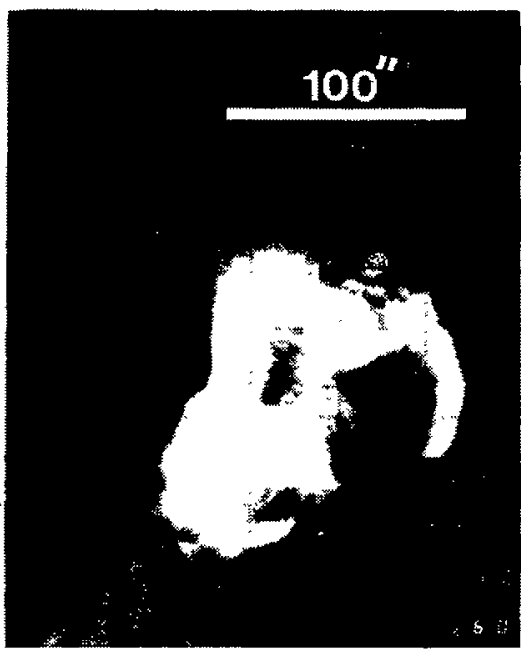

(c)

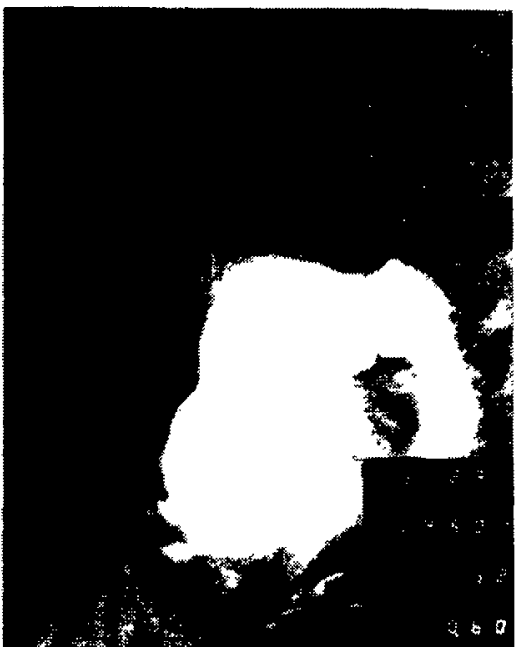

Fig. 1. H $\alpha$ off-center $0.6 \AA$ pictures of the active region NOAA 6555 on 1991 March 22 showing (a) precursors (22:43 UT), (b) remote brightening (22:44UT), and (c) the maximum phase (22:45 UT). An $\mathrm{H} \alpha$ cloud as large as $\approx 100^{\prime \prime} \times 100^{\prime \prime}$ is seen from 22:45 UT to 23:10 UT, after which it diminishes gradually.

was measured to be $\approx 1900 \mathrm{~km} \mathrm{~s}^{-1}$. If we extrapolate the shock passage back to the flare active region under the assumption of a uniform shock speed, we find that the shock started at $\approx 22: 43: 46$ UT. This actually precedes the time of maximum of the microwaves and the $\gamma$-ray continuum (22:44:10-22:44:12 UT; see below) by $\approx 25 \mathrm{~s}$. This result for the relative timing of the shock waves is quite similar to those found in the study of the 1990 May 24 flare (Kocharov et al., 1994).

The microwave bursts during this flare were observed by the Solar Array at OVRO in the frequency range of 1-18 GHz. The most characteristic point of this event is its spectral variation which we show in Figure 3. In the initial rise phase (Figure 3(a)), a spectral peak shows up first at high frequencies and then subsequent enhancement of flux follows up at lower frequencies until it leads to an almost flat spectral shape at the maximum phase. In the decay phase (Figure 3(b)), a gradual softening of the spectrum follows to make the spectral slope more negative with time. While such a spectral activity of microwave fluxes basically carries information on the change of electron energy distribution as well as the magnetic structure of the source, the inversion of the observed flux spectra to electron energy spectra is not straightforward due to the possible dependence of the source area on frequency. For this problem, Lee, Gary, and Zirin (1994) worked out a modelfitting to the observed spectra in which the unusually flat spectral shape and the high level of flux at the maximum phase are used as main model-constraints. The spectral shift from high to low frequencies seen at the rise phase was interpreted either as the expansion of a loop or as the moving up of the microwave-emitting region from a small loop to a large loop (cf., their Figure 6). To compare with the 1990 May 24 microwave bursts, the overall pattern of spectral activities seen 

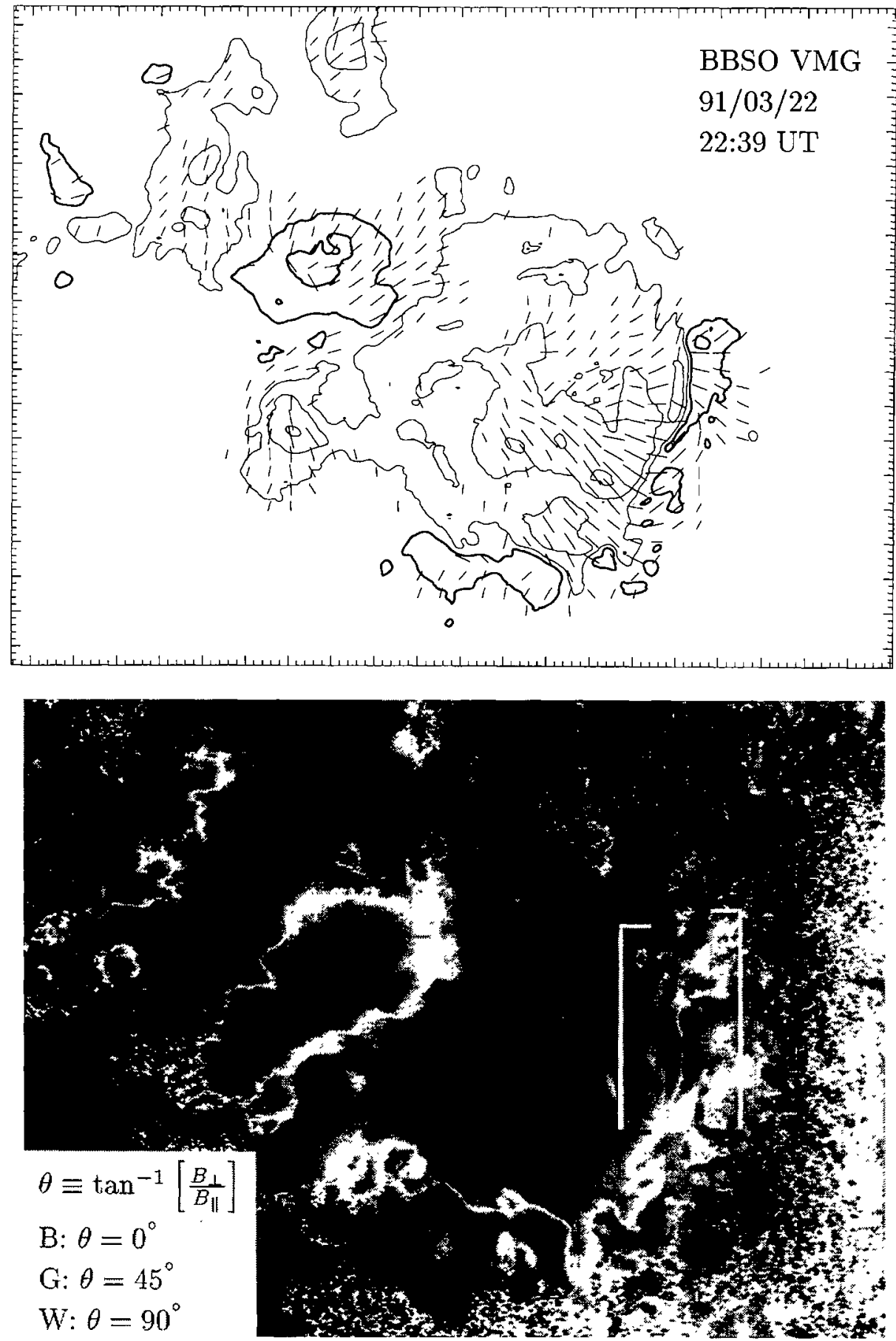

Fig. 2. (a) A vector magnetogram of the active region NOAA 6555 taken at 22:39 UT. Contours represent the strength of the longitudinal fields and the bars represent the direction and the strength of transverse fields. (b) Distribution of inclination angle $(\theta)$ of magnetic fields; $\theta$ varies from $\theta=0^{\circ}$ in the dark region to $\theta=90^{\circ}$ in the white region. The gray color corresponds to $\theta=45^{\circ}$. The bracket in (b) is to locate the region of the He D3 brightening, where $\theta$ is found to lie between $45^{\circ}$ and $90^{\circ}$.

at both flares is similar in the sense that low-frequency fluxes last longer than high-frequency fluxes (see Figure 4). The time scale of the spectral softening of the 1991 March 22 flare is, however, shorter than that of the 1990 May 24 flare. The flux spectra at the maximum phase of both flares appear to be almost the same. 
Accordingly, a model loop with the same parameters was considered in Lee, Gary, and Zirin's (1994) paper to explain the flat and high spectrum seen at the maximum phase. In their results, the source is a big magnetic loop spanning a wide range of magnetic fields from $1500 \mathrm{G}$ down to a few $\mathrm{G}$ in which $N(E>10 \mathrm{keV}) \approx 3 \times 10^{38}$ of highly energetic electrons with energy spectral index of $s_{e} \approx 3$ are trapped. We note that the presence of such a big loop with low field strength is favorable for explaining the presence of a slow neutron injection component with a decay time scale of about $\approx 200 \mathrm{~s}$ (cf., Kocharov et al., 1994).

\section{Neutron and Gamma-Ray Emissions}

Increase of neutron counting rate during 1991 March 22 has been recorded in the Haleakala NM (Pyle and Simpson, 1991; Muraki et al., 1993). In Figure 5 we show the neutron counting rate averaged over 2 and $4 \mathrm{~min}$. Pyle and Simpson (1991) and Muraki et al. (1993) made a fit to these data under the assumption of an impulsive neutron injection from the Sun. They proposed that the injection time coincides with the time of the maximum of $\gamma$-ray continuum and microwave emissions, which is 22:44:10 UT. In the present study, we consider various alternative ways of fitting to the observed neutron counting rates based on our earlier study on the neutron emission of the 1990 May 24 flare (Kocharov et al., 1994). For the parameter fit to the NM data of 1990 May 24, we postulated the presence of two components of neutron injection. The first ( $f$ - or fast) injection component is nearly impulsive with a decay time of $\approx 20 \mathrm{~s}$ and peaks at the time of $\gamma$-ray continuum and microwave emission maximum. The second ( $s-$ or slow) injection component had a longer decay time of $\approx 200 \mathrm{~s}$. Such an idea of the two component injection was motivated to simultaneously account for the impulsive burst at 57$110 \mathrm{MeV}$ and the prolonged one at $2.2 \mathrm{MeV}$ as observed during 1990 May 24 in the GRANAT/PHEBUS experiment. It is essential that this idea was supported by the independent analysis of microwave data too.

Consider the available $\gamma$-ray data for the 1991 March 22 event. According to the GRANAT/PHEBUS experiment, the duration of the main peak over its halfmaximum intensity is $10-15 \mathrm{~s}$ at $10-22 \mathrm{MeV}$ (Vilmer et al., 1994). At 420$600 \mathrm{keV}$, three sub-peaks are seen clearly at 22:43:40-22:44:30 UT with a duration time of 5-20 s each. At $\approx 1 \mathrm{MeV}$, the intensity of the pulse is slightly higher than that of 1990 May 24, while the total duration of the impulsive part of the event is $\approx 4$ times shorter. At $1-4 \mathrm{MeV}$, the $\gamma$-ray continuum spectrum is softer than that of 1990 May 24. As a result, the maximum intensity at $10 \mathrm{MeV}$ is weaker than that of 1990 May 24 by a factor of $\approx 4$. Similar to the case of the 1990 May 24 flare, some hardening of the spectrum at high energies is observed after the time of the maximum of the bursts. A spectral hardening in this energy range is usually attributed to the contribution from the $\pi^{0}$-decay emission. As in the 1990 May 24 event, a strong $2.2 \mathrm{MeV}$ line emission is observed in the decay phase of the event 


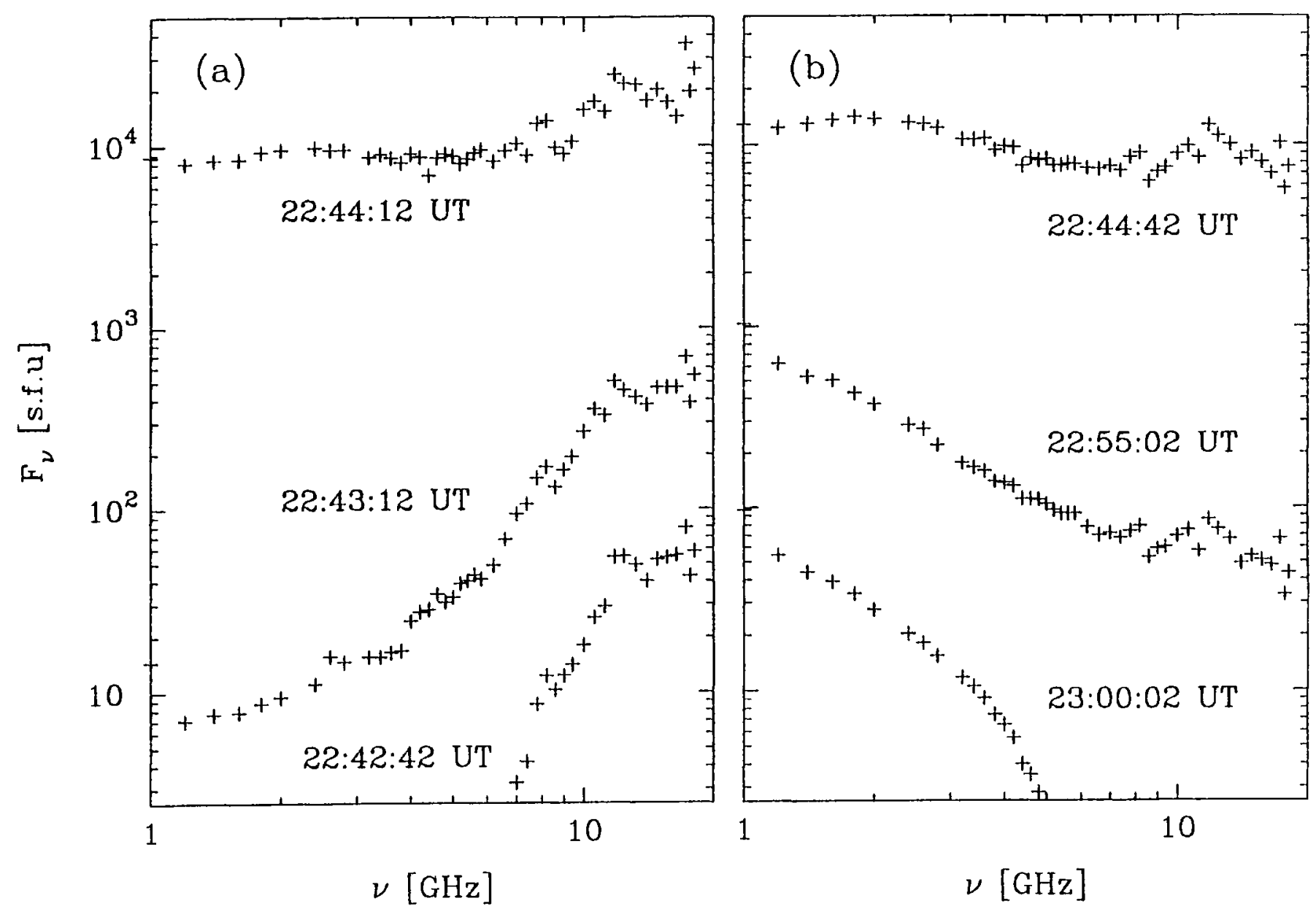

Fig. 3. The spectral activity of the 1991 March 22 microwave bursts: (a) the initial rise phase and the maximum phase and (b) the decay phase. 
1991 March 22

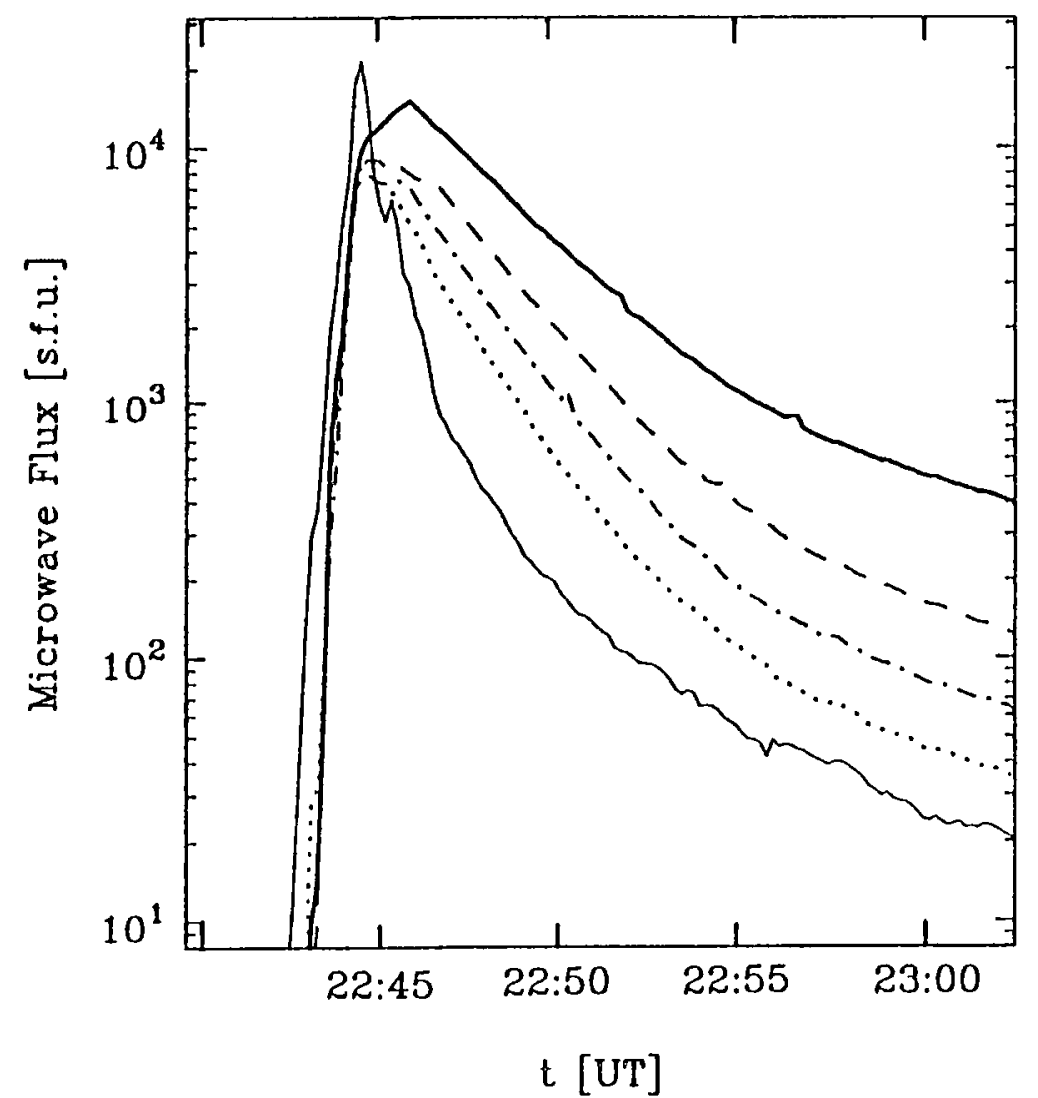

1990 May 24

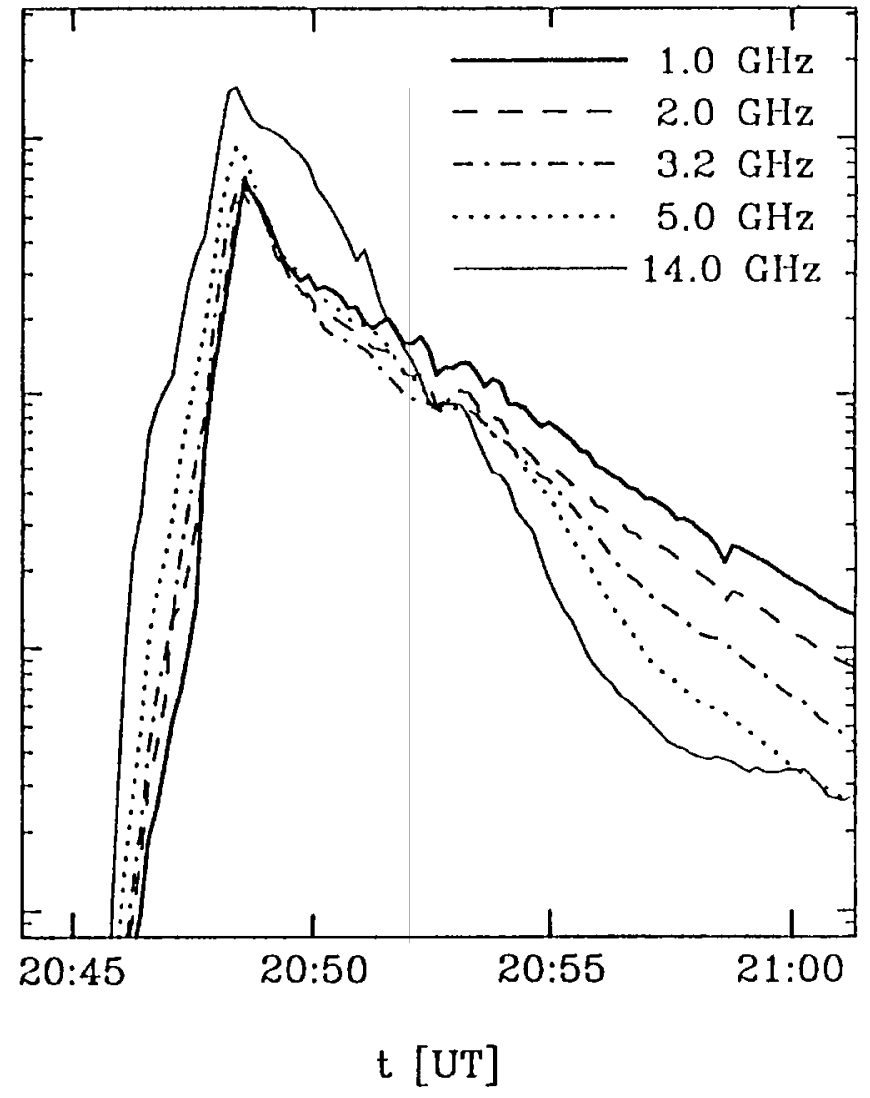

Fig. 4. Multifrequency time-profiles of the microwave bursts during (a) the 1991 March 22 flare and (b) the 1990 May 24 flare. 
after 22:44:20 UT. A difference between those two events is that a high-energy $(>10 \mathrm{MeV})$ tail above a significant level was detected during the 1990 May 24 flare, while such a high-energy tail was absent during the 1991 March 22 flare (Talon et al., 1993; Vilmer et al., 1994). Unfortunately, all data published so far regarding the nuclear $\gamma$-ray emission during the 1991 March 22 flare are rather qualitative. For the purpose of a quantitative estimation, we introduce a few assumptions. In view of the report of the strong bursts at $2.2 \mathrm{MeV}$, we may consider that the value of $2.2 \mathrm{MeV}$ line fluence, $F(2.2 \mathrm{MeV})$, of $1991 \mathrm{March} 22$ should, at least, amount to $\geq 0.2$ of that of 1990 May 24 . Note that the fluence of the $2.2 \mathrm{MeV}$ line in the 1990 May 24 flare was $F(2.2 \mathrm{MeV})=345 \mathrm{~cm}^{-2}$ (Terekhov et al., 1993). From the absence of a significant high-energy tail in the 1991 March 22 bursts, we assume that the fluence of $>10 \mathrm{MeV} \gamma$-rays is $\leq 0.2$ of that on 1990 May 24 .

With currently available $\gamma$-ray data of the 1991 March 22 flare, it appears hard to set one particular scenario for the neutron injection, unlike in our previous study of the 1990 May 24 flare. For this reason, we consider various possibilities of fitting the observed NM counting rate of the 1991 March 22 flare. These include (1) two impulsive components of neutron injection, in view of the multi-impulsive nature of the $\gamma$-ray burst time profiles from the GRANAT, and (2) two components with a combination of the 'impulsive-plus-prolonged' injection of neutrons similar to the case of the 1990 May 24 flare. Under these scenarios of neutron injection, we model the neutron injection function as

$$
N_{n}(E, t)=N_{f} \exp \left[-\frac{E}{E_{f}}-\frac{t}{T_{f}}\right]+N_{s} \exp \left[-\frac{E}{E_{s}}-\frac{t}{T_{s}}\right]
$$

in units of [ $\mathrm{sr}^{-1}$, where $N_{f(s)}, E_{f(s)}$, and $T_{f(s)}$ denote number, energy spectrum, and decay time of the first (second) injection component respectively.

The resulting NM counting rate, $I_{N M}(t)$, is then given by

$$
I_{N M}(t)=\frac{1}{R^{2}} \int_{E_{t h}}^{\infty} N_{n}\left(E, t-\frac{R}{v}\right) S(E) \exp \left[-\frac{R}{\gamma \tau v}\right] \mathrm{d} E,
$$

where $E, v$, and $\tau$ refer to the energy, the velocity, the Lorentz-factor and the decay time a neutron, respectively. In this expression, $S(E)$ is the NM response function for solar neutrons; Eth is the threshold energy of the NM; $R=1$ AU. $S(E)$ depends on the type of NM used with, its altitude and the zenith angle. We obtain $S(E)$ by using the software developed by Efimov and Terekhov (1988) which takes into account the propagation of solar neutrons through the Earth's atmosphere. We compare the $I_{N M}(t)$ calculated using (2) with the Haleakala NM counting rates over the four time bins: $22: 44-46,22: 46-48,22: 48-52$, and $22: 52-56$ under the $\chi^{2}$-test (see Figure 5). The data at the last time bin, 22:56-23:00 UT, is used as an additional criterion for the upper limit. In the present simulation, we fix $T_{f}=20 \mathrm{~s}$ and vary the values of $E_{f}, E_{s}, T_{s}$, and the ratio of the $f$-to-s components above 

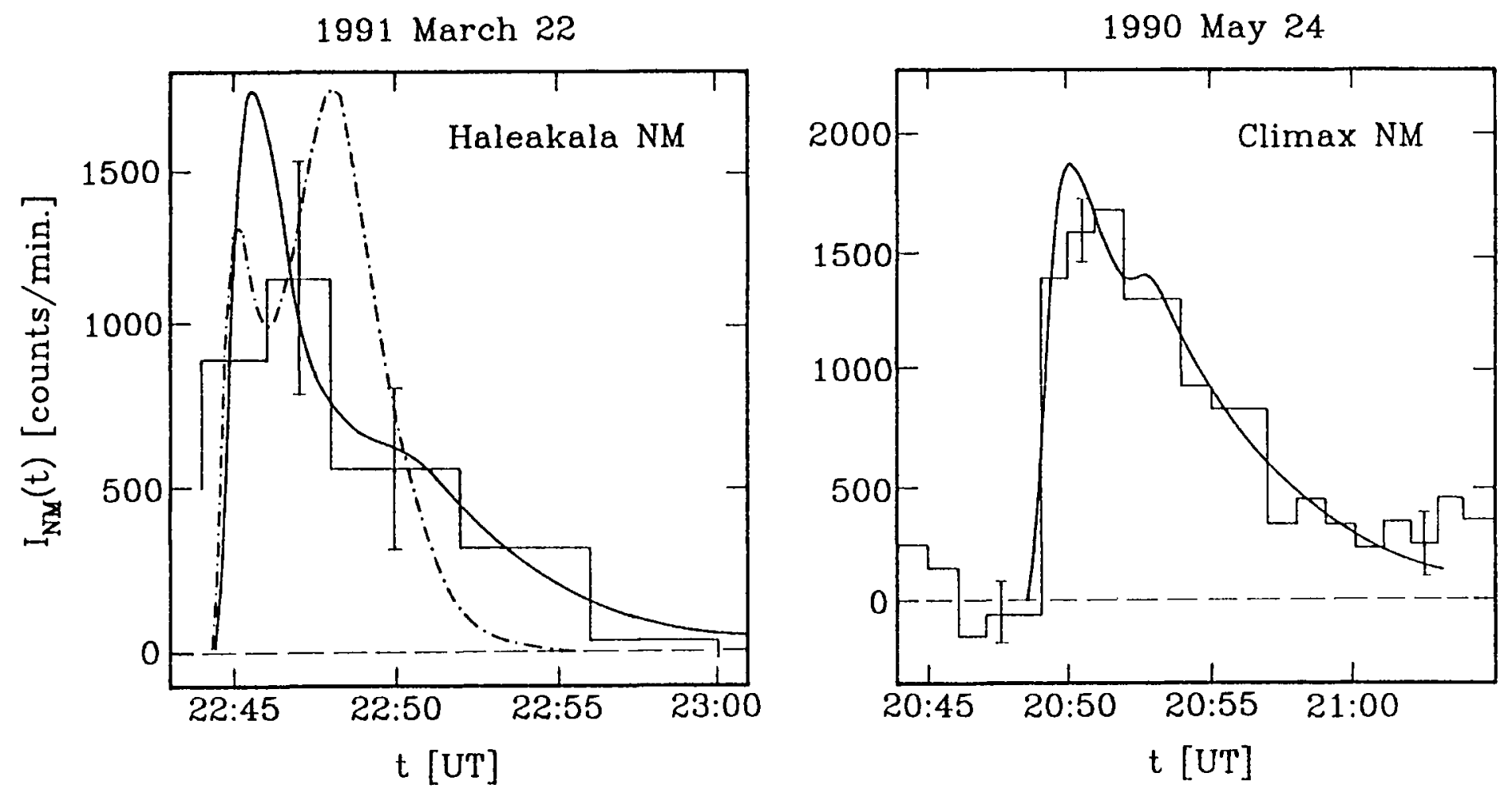

Fig. 5. Fitting to the NM counting rate. (a) The Haleakala NM counting rate, $I_{N M}(t)$, on 1991 March 22 (Pyle and Simpson, 1991) vs the calculated $I_{N M}(t)$ in the present study; the solid curve is the the result of Case 2a and the dot-dashed curve is that of Case 1. (b) The Climax NM counting rate on 1990 May 24 vs the calculated $I_{N M}(t)$ (the solid curve) made in the paper by Kocharov et al. (1994). 
$300 \mathrm{MeV}, X=N_{f}(>300 \mathrm{MeV}) / N_{s}(>300 \mathrm{MeV})$, until a satisfactory agreement to the observed NM data is obtained. Note that the precise value of $T_{f}$ is not essential for the present parameter fit to the NM data as long as it is small enough. The resulting best-fit parameters in each case are presented in Table I together with the $\chi^{2}$ value of each fitting.

In addition to finding out the best-fit parameters, it also appears important to calculate the fluence of the $2.2 \mathrm{MeV} \gamma$-ray line emission expected in each case. It is known that the $2.2 \mathrm{MeV}$ line fluence correlates well with the total number of $30 \mathrm{MeV}$ neutrons produced at the Sun in all directions (see Kocharov et al., 1994). We use this fact and Hua and Lingenfelter's (1987) results to calculate the fluences of $2.2 \mathrm{MeV}$ emission from the $f$ - and the $s$-components, respectively. We represent the fluence as $F^{0}(2.2 \mathrm{MeV})=5 \times 10^{-26} Q_{n}(30 \mathrm{MeV}) /(4 \pi)$, where $Q_{n}(30 \mathrm{MeV})$ is the total number of $30 \mathrm{MeV}$ neutrons generated in all directions (in $\mathrm{MeV}^{-1}$ ). The numerical coefficient in this expression takes into account the heliocentric position of the 1991 March 22 flare - such a dependence on the position of the flare is due to the absorption of the $2.2 \mathrm{MeV}$ emission. In practice, the neutron flux observed at the Earth is used to calculate $F^{0}(2.2 \mathrm{MeV})$. The $2.2 \mathrm{MeV}$ line fluence obtained in this way corresponds to the case of the isotropic generation of neutrons. Our results of the fluence from each component, $F_{f}^{0}(2.2 \mathrm{MeV})$ and $F_{s}^{0}(2.2 \mathrm{MeV})$, as well as the number of neutrons injected towards the Earth, $N_{n}(>300 \mathrm{MeV})$, are presented in the last three columns of Table I. In case of the anisotropic generation of high-energy neutrons, we have to multiply these fluences by the anisotropy factor, $A_{n}$, as $F(2.2 \mathrm{MeV})=A_{n} F^{0}(2.2 \mathrm{MeV})$. Here $A_{n}$ is the anisotropy factor for high-energy neutrons, which is given by

$$
A_{n}=\frac{Q_{n}(>300 \mathrm{MeV})}{4 \pi N_{n}(>300 \mathrm{MeV})},
$$

where $Q_{n}(>300 \mathrm{MeV})$ is the total number of $>300 \mathrm{MeV}$ neutrons generated in all directions and $N_{n}(>300 \mathrm{MeV})$ is the number of neutrons propagating in the Earth's direction per unit solid angle. Note that theoretical values of $A_{n}$ vary from $\approx 1$ to $\approx 1000$ (see Section 4.1).

The results of the parameter fit and the calculation of $F^{0}(2.2 \mathrm{MeV})$ and $N_{n}(>300 \mathrm{MeV})$ are summarized as follows:

In Case 1, we take $T_{s}=T_{f}=20 \mathrm{~s}$, i.e., two impulsive components of neutron injection. At $80 \%$ probability, $E_{s}=70-140 \mathrm{MeV}$ and $E_{f} \geq 400 \mathrm{MeV}$. We note that one impulsive component of neutron injection with a power-law energy spectrum can also give a satisfactory fit with the choice of the power-law index of $s_{n}=2.9 \pm 0.9$ (at $90 \%$ probability) and the cut-off energy at $>1.5 \mathrm{GeV}$. In any case, the total number of $>300 \mathrm{MeV}$ neutrons injected towards the Earth comes out to be nearly the same: $N_{n}(>300 \mathrm{MeV})=(2-3) \times 10^{27} \mathrm{sr}^{-1}$, inside the $80 \%$ confidence contours. To verify the assumption of impulsive neutron emission, the 2.2 MeV and other nuclear $\gamma$-ray line emissions are needed. For the exponential 


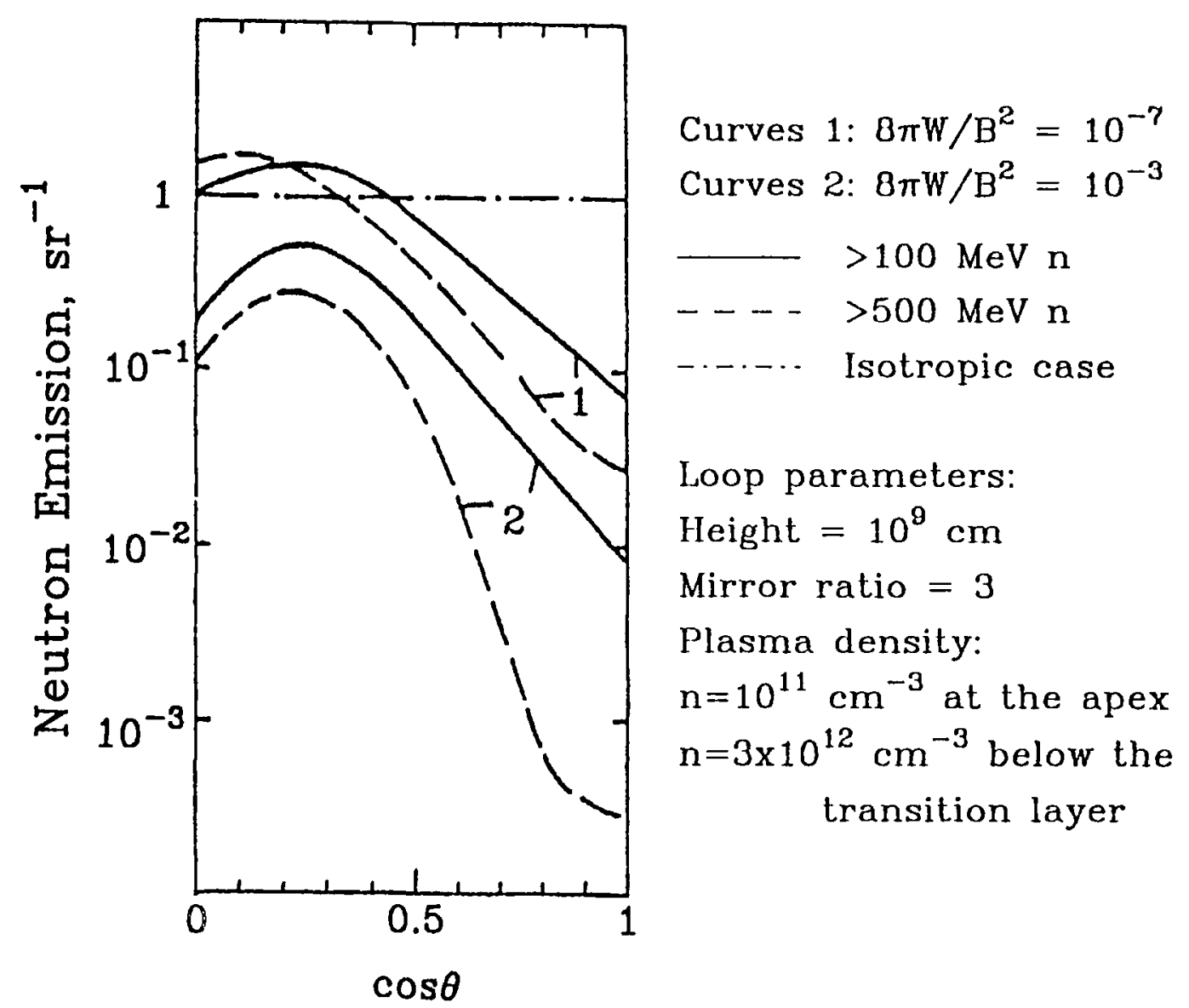

Fig. 6. Calculated neutron emission from the Sun as a function of $\cos \theta$ for cases of weak pitch-angle scattering (curves 1 ) and moderate/strong pitch-angle scattering (curves 2 ). $\theta$ is the angle between the line of sight and the magnetic fields in the footpoints of the magnetic loop. The dashed lines are for $>500 \mathrm{MeV}$ neutrons and the solid lines, for $>100 \mathrm{MeV}$ neutrons. Dash-dotted line is for isotropic case. $W$ is the MHD turbulence energy density. Loop parameters are also specified. This calculation is made for magnetic fields in a loop leg standing normal to the solar surface (see, for details, Gueglenko et al., 1990).

energy spectrum of neutrons, the predicted fluence of the $2.2 \mathrm{MeV}$ line emission is $F^{0}(2.2 \mathrm{MeV})=(5-50) A_{n} \mathrm{~cm}^{-1}$.

In Case 2a, we make use of the scenario similar to that deduced for the 1990 May 24 flare. very wide range of values of $E_{s}$ and $E_{f}$ is possible. However, if we require In this case, $F^{0}(2.2 \mathrm{MeV}) \geq 1 \mathrm{~cm}^{-2}$, the possible range of $E_{s}$ and $E_{f}$ is narrowed to $50-200 \mathrm{MeV}$ and $200-560 \mathrm{MeV}$, respectively. Inside the $80 \%$ confidence contour, values $F(2.2 \mathrm{MeV}) \leq 250 A_{n} \mathrm{~cm}^{-2}$ are possible. In addition, under the same scenario, we consider Case $2 b$ and $2 c$ as well (see Table I). We note that the $2.2 \mathrm{MeV}$ line fluences predicted in Cases $2 \mathrm{a}, \mathrm{b}, \mathrm{c}$ lies in so wide a range that any observed value of $F(2.2 \mathrm{MeV})$ can possibly be covered.

In Figure 5, we compare the calculated $I_{N M}(t)$ in Case 1 and Case 2a with the Haleakala NM data. For comparison, we also show, in Figure 5, our previous parameter fitting to the Climax NM data of 1990 May 24. The present result of Case 2a looks very similar to the fitting result for the 1990 May 24 flare. Even 
an excess of the calculated counting rate over the observed counting rate around the onset time is commonly seen in both events. Note, that to remove this excess, a three-component neutron injection may be proposed. Regarding which case is more plausible, the detailed information of the $2.2 \mathrm{MeV}$ line emission is important because its fluence, decay time, and the time of maximum intensity predicted in each case makes a big difference. A quantity which might be determined by comparison of the fluence $F^{0}(2.2 \mathrm{MeV})$ with the real observation is the anisotropy factor (see Equation (3)). Recall that we recently obtained $A_{n} \approx 3$ for the 1990 May 24 limb flare using this method (Kocharov et al., 1994).

\section{Anisotropy of High-Energy Secondary Emissions}

We first summarize the general predictions for the high-energy secondary emissions in Section 4.1. We then consider the specific value of the anisotropy factor of the emissions during the 1991 March 22 flare in Section 4.2.

\subsection{THEORETICAL PREDICTIONS}

The anisotropy of the secondary emission has been discussed both on purely theoretical grounds or under empirical considerations. Theoretically, the secondary emission is expected to be more concentrated to the angle normal to the magnetic field in the loop 'legs' although the detailed angular distribution depends on the rate of pitch-angle scattering by MHD waves (see Mandzhavidze and Ramaty, 1992, and references therein). In Figure 6, we show a result for the angular distribution of neutron emission obtained for different scattering regimes. The neutron emission in this figure is normalized to the isotropic case so that one can easily read off the anisotropy factor. In general, the anisotropic neutron flux falls below the isotropic flux as $\theta$ decreases. Note that in Figure 6 the anisotropic flux falls below the isotropic flux also at $0 \leq \cos \theta \leq 0.2$. This is due to the scattering of escaping neutrons that happens if the 'legs' of the loop are vertical only. We note however that, for a wide range of the pitch-angle scattering, the emission generated roughly normal to the magnetic field does not differ more than by a factor of $\approx 3$ from the isotropic value. On the other hand, the emission generated upwards along the magnetic field in the loop 'leg' may be depressed by 2-3 orders of magnitude. In this way, the angle, $\theta$, appears to be the most important parameter in theoretical prediction for the anisotropic factor of high-energy emission, although the degree of pitch-angle scattering is yet another factor to be specified.

An empirical way of determining the anisotropy of high-energy secondary emission is the statistical analysis of heliolongitude distribution of flares accompanied by high-energy $\gamma$-ray emission (Vestrand et al., 1987). In the 21st cycle of solar activity strong limb brightening of $>10 \mathrm{MeV} \gamma$-ray emission was observed. Small hardening of $0.3-1 \mathrm{MeV}$ emission towards the limb was also reported by Vestrand 
et al. (1987). Kocharov et al. (1991) presented evidence of limb brightening of highenergy neutron emission detected by various NMs. There is thus evidence for the anisotropy of high-energy secondary emission (for the review see Mandzhavidze and Ramaty, 1993). Meanwhile, a rather small limb brightening of $>10 \mathrm{MeV}$ $\gamma$-rays was seen in the 22nd cycle (Vestrand et al., 1991). Furthermore, Kocharov et al. (1991) noted that, during the 21st cycle, a number of powerful flares with 4-7 MeV nuclear $\gamma$-ray line emission were randomly located closer to limb too, although Kocharov and Kovaltsov (1990) calculated the possible anisotropy factor of nuclear $\gamma$-ray line emission to find that it is no more than a factor of 1.4. Hence, the statistical approach bears special uncertainties and needs to be complemented by some other methods of determining the anisotropy.

A $\gamma$-ray spectrum at $100 \mathrm{keV}-10 \mathrm{MeV}$ can be used to determine the anisotropy factor if it is due to electron bremsstrahlung. In the isotropic case, a power-law electron spectrum produces a nearly power-law bremsstrahlung spectrum in this energy range (Ramaty et al., 1994). The possible anisotropy of the column density traversed by the particles is an essential factor that leads to the anisotropic radiation. For the disk flare, this effect may lead to an essential steepening of the $\gamma$-ray spectrum above $0.5 \mathrm{MeV}$ (i.e., at electron Lorentz-factor $\gamma>2$ ). On the other hand, at very high energies the angular width of the instantaneous emission of each electron is much less than the angular width of the column density distribution and than the angular width of the electron distribution function. Hence, further narrowing of the instantaneous emission of each electron leads to no change in the emission of the ensemble of particles integrated over some reasonable time interval. As a result, the slope of the $\gamma$-ray spectrum at $\gamma \gg 1$ is nearly the same as in the isotropic model, but the total intensity differs by the anisotropy factor, $A_{b r}$, from that of the isotropic case. The characteristic width of angular distribution of the column density is $\approx \frac{1}{4} \mathrm{rad}$ (Kocharov and Kovaltsov, 1990). Thus, at $\gamma>10$, the electron energy is high enough to have little influence on the slope of the bremsstrahlung spectrum. In this case, the value of $A_{b r}$ (averaged over the real magnetic fields) can be obtained from the difference between the powerlaw spectrum extrapolated from the region below $500 \mathrm{keV}$ to the region above $5 \mathrm{MeV}$ and the observed spectrum. For this reason the energy band $1-5 \mathrm{MeV}$ is most adequate for the study of the anisotropy of electron bremsstrahlung although the synchrotron loss at ultrarelativistic energies may lead to a steepening of the bremsstrahlung spectrum introducing some overestimation of $A_{b r}$.

As a practical way to measure the anisotropy factor, $A_{n}$, from the neutron monitor data, we would like to stress the following: as shown in Table I, the number of $>300 \mathrm{MeV}$ neutrons, $N_{n}(>300 \mathrm{MeV})$, emitted in Earth's direction required to fit the observed NM counting rate is almost the same for all cases considered. This is simply a result of the given shape of the NM response function. On the other hand, it can be shown that the generation of $\pi^{0}$-decay emission correlates well with that of $>300 \mathrm{MeV}$ neutrons. The calculated ratio of total generation rate of $>300 \mathrm{MeV}$ neutrons to that of $\pi^{0}$-decay $\gamma$-rays in $10-100 \mathrm{MeV}$ 
range is $Q_{n}(>300 \mathrm{MeV}) / Q_{\gamma}\left(\pi^{0}\right) \approx 2.2$ for the power-law index of primary proton spectrum $s_{p}=2-6$. Therefore, the measured fluence of $\pi^{0}$-decay $\gamma$-ray emission, $F\left(\pi^{0}\right) \approx Q_{\gamma}\left(\pi^{0}\right) /\left(4 \pi R_{a}^{2}\right)$, can be used to calculate the total number of high-energy neutrons produced in all directions. We then obtain the anisotropy factor, $A_{n}$, from the NM data under Equation (3). It is necessary to note that using $\pi^{0}$-decay emission we might overestimate $A_{n}$ by a factor of up to 2 , because of a weak anisotropy of $\pi^{0}$-decay emission itself, which can however be corrected to some extent. This approach is better than the method in which the $2.2 \mathrm{MeV} \gamma$-ray line emission is used. Since it is the low-energy ( $30 \mathrm{MeV})$ neutron production that the $2.2 \mathrm{MeV}$ emission has a good correlation with, this method requires the extrapolation of the neutron energy spectrum over an order of magnitude in energy, which may introduce greater uncertainty.

\subsection{THE 1991 MARCH 22 SOLAR FLARE}

To estimate the anisotropy factor of the neutron emission of the 1991 March 22 flare, we may use the inclination angles over the flaring region as determined from the vector magnetogram (Figure 2) in the theoretical model results shown in Figure 6. Identifying the position of the He D3 brightening with the source of the fast component, we find, from Figure 2, that the inclination angles in this region appear in the range of $45^{\circ} \leq \theta \leq 90^{\circ}$. From Figure 6, we then expect that the anisotropy factor is $\leq 10$ in the case of weak pitch-angle scattering and $\leq 100$ in the case of moderate/strong pitch-angle scattering. If an average is taken over the distribution of the inclination angle, $\theta$, over this region, we may expect that the anisotropy factor is, at most, $\approx 1-10$.

The above estimation of the anisotropy is also valid for the bremsstrahlung radiation. In addition, we can also estimate the anisotropy factor from the bremsstrahlung spectrum as illustrated in Section 4.1. If we use the electron distribution with a power-law index of $s_{e}=3$ (see Section 2) in the traditional isotropic thick-target model, we can expect a nearly power-law bremsstrahlung spectrum with the spectral index, $s_{\gamma}$, which gradually increases from $s_{\gamma} \approx 1.8$ at $<0.5 \mathrm{MeV}$ to $s_{\gamma} \approx 2.2$ at 5-10 MeV (Ramaty et al., 1993, 1994). According to our preliminary analysis, the GRANAT observations made in the lowest and the highest energy bands at the time of the maximum intensity (Vilmer et al., 1994; Pelaez, 1993) do not contradict our prediction for the spectral slopes. We also note that the observation shows evidence of the spectral steepening at several $\mathrm{MeV}$. If we extrapolate the power-law distribution with $s_{\gamma}=1.8$ at $420-600 \mathrm{keV}$ into $10-22 \mathrm{MeV}$, the extrapolated counting rate in this energy regime exceeds the observational data of the GRANAT/PHEBUS by a factor of $\approx 5$. This amount of excess may be attributed mainly to the directivity of the electron bremsstrahlung that results in the additional steepening of the $\gamma$-ray spectrum at several MeV. Accordingly, the directivity of electron bremsstrahlung, $A_{b r}$, is also not very large, at most, $A_{b r}<10$. This 
conclusion is in a good agreement with the above-mentioned result for the degree of anisotropy based on the inclination angles of the magnetic fields.

We thus conclude that, in both cases, the anisotropy factor of the impulsive part (the $f$-component) of high-energy secondary emission would be not very high, but $1 \leq A_{n(b r)} \leq 10$. For the source of the slowly decaying component, we considered a loop with length $\approx 2 \times 10^{10} \mathrm{~cm}$. Without imaging of the microwave source, we cannot specify particular values for the inclination angles of the magnetic fields in the 'legs' of this loop. In general, it is likely that they will span a wider range because of a bigger cross section expected for the slow component as compared with that of the small loop of the fast component. In this sense, any theoretical value of $A_{n}$ ranging from $\approx 1$ to $\approx 1000$ is possible for the $s$-component of the emission. However, the fact that a strong tail of the $>10 \mathrm{MeV} \gamma$-ray emission from $\pi^{0}$-decay was absent in this event limits the possible value of $A_{n}$ for the slow component too.

We can calculate the fluence of the $\pi^{0}$-decay emission, $F\left(\pi^{0}\right)$, using the fact that $\pi^{0}$-decay emission correlates well with the emission of high-energy neutrons. In Case 1, where the two impulsive components of neutron injection were considered, the predicted fluence of $10-100 \mathrm{MeV}$ emission from $\pi^{0}$-decay is $F\left(\pi^{0}\right)=(0.07-1) F_{24}\left(\pi^{0}\right)$. Here $F_{24}\left(\pi^{0}\right)$ denotes the fluence of $10-100 \mathrm{MeV}$ $\pi^{0}$-decay emission corresponding to the total number of high-energy neutrons $Q_{n}(>300 \mathrm{MeV}) /(4 \pi)=3 \times 10^{28} \mathrm{sr}^{-1}$ which we regard as the fluence of the tail of the 1990 May $24 \gamma$-ray event observed by Talon et al. (1993). The $2.2 \mathrm{MeV}$ line fluence predicted in this case is in the range of $5-570 \mathrm{~cm}^{-2}$ and, for the best-fit parameters, it is $F(2.2 \mathrm{MeV})=15-150 \mathrm{~cm}^{-2}$. Note that a higher value of $F(2.2 \mathrm{MeV})$ corresponds to a higher value of $F\left(\pi^{0}\right)$. In Case 2, the fluence comes out as $F\left(\pi^{0}\right)=A_{n} F_{24}\left(\pi^{0}\right) / 25($ Cases $2 \mathrm{a}, \mathrm{c})$, and $F\left(\pi^{0}\right)=A_{n} F_{24}\left(\pi^{0}\right) / 64$ (Case $2 \mathrm{~b}$ ). Thus the fluence of the high-energy tail can be low enough to satisfy the constraint, $F\left(\pi^{0}\right) \leq 0.2 F_{24}\left(\pi^{0}\right)$, under the choice of a moderate anisotropy factor, $A_{n} \leq 5-13$, for the slow component.

To compare with the 1990 May $24 \gamma$-ray bursts, we note that the total duration of the main peak of the $\gamma$-ray continuum of 1991 March 22 was $\approx 4$ times shorter than that of 1990 May 24, and that the tail of high-energy ( $>10 \mathrm{MeV}) \gamma$-rays of 1991 March 22 was $\geq 5$ times lower than that of 1990 May 24. Thus, one can expect the flux of $\pi^{0}$-decay emission of 1991 March 22 should be lower than that of 1990 May 24. On the other hand, it is the disk position of the 1991 March 22 flare that can lead to a lower absorption of the $2.2 \mathrm{MeV}$ emission compared with the 1990 May 24 flare (cf., Hua and Lingenfelter, 1987). As a result, a similar level of the $2.2 \mathrm{MeV}$ line fluence can be produced although the number of neutrons is smaller by a factor of 4 . For these two reasons we presume that the total number of neutrons generated in all directions, $Q_{n}$, during the 1991 March 22 flare would be less than that of the 1990 May 24 flare. Therefore, the smaller number of $>300 \mathrm{MeV}$ neutrons detected during the 1991 March 22 flare is not only due to its location on the solar disk but also due to the smaller number of protons accelerated during 
this event, as compared with the 1990 May 24 flare. Keeping in mind the value $A_{n} \approx 3$ obtained for the 1990 May 24 flare, we feel that the most likely value of the anisotropy factor for the 1991 March 22 flare would be $A_{n} \approx 5-10$.

\section{Discussion}

Our analysis of the NM counting rate and the $\gamma$-rays of the 1991 March 22 flare has been based on the postulation of a two-component neutron injection, as in the study of the 1990 May 24 flare, which is motivated by noting the similarity of the microwave activities of both flares. Besides such a similarity between two flares, the 1991 March 22 flare showed several other properties different from those of the 1990 May 24 flare and other flares originating from the same active region. Wc discuss them in order to further clarify the nature of the 1991 March 22 flare.

The high-energy secondary emission of the 1991 March 22 flare is, to some extent, more impulsive than that of the 1990 May 24 flare. The microwave spectral activity also implies a longer time scale for the electron acceleration in the 1990 May 24 event, in view of the fact that the high-frequency component (impulsive component) of this event shows a longer half-life time than that of the 1991 March 22 flare (see Figure 4). In both events, however, it appears that the acceleration/deceleration took place at a time scale no longer than several minutes. It is known that impulsive solar flares are usually accompanied by low fluxes of energetic protons in the interplanetary medium (Kocharov et al., 1983; see, for a review, Ramaty et al., 1993). The 1991 March 22 flare is, however, not this case. According to the observations of solar energetic particles aboard the GRANAT satellite (Ermakov et al., 1993), the flare was accompanied by one of the greatest increases of protons and $\alpha$-particles. The maximum proton flux at $1.3-2.5 \mathrm{MeV}$ exceeded $10^{4} \mathrm{~cm}^{-2} \mathrm{~s}^{-1} \mathrm{sr}^{-1} \mathrm{MeV}^{-1}$. There occurred several big flares in the same active region of 1991 March 22, including the 1991 March 25 flare (X5.3/3B, S24 W13, 08:18 UT), the 1991 March 26 flare (X4.7/3B, S28 W23, 20:34 UT) and the 1991 March 29 flare (X2.4/3B, S28 W60, 06:48 UT). However, no significant increase in solar cosmic rays has been detected for these flares (Solar Geophysical Data, 1991, No. 560, part 1). As a possible factor that can lead to such a dissimilarity, we consider an additional acceleration that took place at a distance from the 'core' of the 1991 March 22 flare to produce the large solar energetic particle event, without a significant increase in the secondary emission. According to Wang et al. (1994), a local magnetic flux emerged during the flare and it led to a significant change of magnetic shear angles and a more spacious development of the flare. The observed spectral shift of microwave flux from low to high frequencies (Figure 3) and the expansion of $\mathrm{H} \alpha$ brightening from the core to a remote region (Figure 2) may be considered as the observational signatures of this process. After the 1991 March 22 flare, a shock wave with velocity $\approx 1400 \mathrm{~km} \mathrm{~s}^{-1}$ was observed in the interplanetary medium. Energetic particles with energy $2-3 \mathrm{MeV} \mathrm{n}^{-1}$ detected before the 
arrival of the shock were enriched by helium: $\mathrm{He} / p \approx 0.2$. This helium abundance is typical for an impulsive flare. Ermakov et al. (1993) consider it as evidence for particle acceleration in the chromosphere or in the lower corona of the Sun. The observed decay times of soft X-ray emission ( $<10 \mathrm{~min})$ and the total duration of the bursts of hard $\mathrm{X}=/ \gamma$-rays $(\sim 2 \mathrm{~min})$ during 1990 May 24 and 1991 March 22 are typical for impulsive solar flares. The spectral index of microwave flux at $9 \mathrm{GHz}$ is close to zero, which corresponds to a class between the impulsive flares and the gradual ones (Kocharov et al., 1991). The solar cosmic ray events accompanying the 1991 March 22 and the 1990 May 24 flares showed different intensities, both of which, however, exceeded that of a typical impulsive flare. All of these results lead to the idea that a flare showing a flat microwave spectrum tends to belong to an intermediate class between a typical impulsive flare and a gradual flare. The reason for the existence of such an intermediate class may be speculated in a case where the energy released during flares has a more or less continuous distribution over a wide range of spatial/time scales of flares. Obviously, a statistical study based on a large sample is needed to verify this hypothesis.

\section{Summary}

In this paper, we have analyzed the Haleakala NM data and the GRANAT/PHEBUS $\gamma$-ray data of the 1991 March 22 flare based on information of macroscopic parameters gathered through the $\mathrm{H} \alpha$ images, magnetograms, and microwave spectra obtained at BBSO and OVRO. The $\mathrm{H} \alpha$ observations showed the presence of small magnetic loops with a length $<3 \times 10^{9} \mathrm{~cm}$ which we regarded as the source of the fast (most impulsive) component of the secondary emission. The high microwave fluxes at low frequencies indicate the presence of a larger loop with a length about $2 \times 10^{10} \mathrm{~cm}$, which is favorable for forming a slowly decaying component of the secondary emission. After making a parameter fit of the neutron injection function to the observed NM counting rate, we concluded that the NM observation can be accounted for either by an impulsive neutron injection or by the combination of 'impulsive-plus-prolonged' components of neutron injection at the Sun. In the first case, the primary accelerated protons would be trapped inside the above-mentioned small loops. In the second case, trapping of protons both in small and large loops is necessary. The latter scenario gives a better fit to NM data and seems more attractive due to the similarity of microwave emissions of the 1991 March 22 and 1990 May 24 solar flares. In an effort to find a more plausible scenario, we carried out the calculation of the fluence of the $2.2 \mathrm{MeV}$ line emission expected in each case. A later investigation of the time profiles of the $2.2 \mathrm{MeV} \gamma$-ray line is thus needed to make a final conclusion.

Under various possible choices of the neutron injection function, the total number of $>300 \mathrm{MeV}$ neutrons injected to the Earth comes out as $2-3 \times 10^{27} \mathrm{sr}^{-1}$, which is much less than the value found for the 1990 May 24 limb flare. We exam- 
ined if the difference in the $>300 \mathrm{MeV}$ neutron flux is due to possibly a high degree of anisotropy of the emission. To estimate the anisotropy factor, we examined the inclination of the magnetic fields at the source obtained from BBSO and also the $\gamma$-ray spectrum obtained from the GRANAT/PHEBUS. It appeared that the large inclination angles of the magnetic fields at footpoints of the impulsive component loops offset, to some extent, the effect of the directivity of high-energy secondary emissions. The fact that a strong tail of the $>10 \mathrm{MeV}$ emission was absent in the event limits the possible value of directivity of slow component too. As a result, the anisotropy factor is not very large, but $A \approx 1-10$, which is much smaller than the theoretically predicted values, as high as, $\approx 100-1000$. We concluded that the smaller number of $>300 \mathrm{MeV}$ neutron flux detected during the 1991 March 22 flare is only partly due to its location on the solar disk, and that the rest of the difference in the neutron number must be due to the smaller number of primary protons accelerated during the 1991 March 22 flare. For a more precise determination of the anisotropy factor for the high-energy neutron emission, observation of the flux of $\pi^{0}$-decay $\gamma$-rays is necessary.

\section{Acknowledgements}

Observations at BBSO have been funded by NASA grant NAGW-1972 and observations at OVRO by NSF grants ATM-9311416 and AST-9314929 to the California Institute of Technology. One of us (LGK) has been supported by NSF grant ATM9122023 during his visit to BBSO.

\section{References}

Bai, T.: 1988, Astrophys. J. 334, 1049.

Cacciani, A., Varsik, J., and Zirin, H.: 1989, Solar Phys. 125, 173.

Efimov, Yu. E. and Terekhov, M. M.: 1988, Geomagn. Aeron. 28, 832 (in Russian).

Ermakov, S. I., Kontor, N. N., Lyubimov, G. P., Pavlov, N. N., Tulupov, V. I., Chuchkov, E. A., and Scherbovski, B. Ya.: 1993, Izv. Akademii Nauk 57, 7 (in Russian).

Gueglenko, V. G., Kocharov, G. E., Kovaltsov, G. A., Kocharov, L. G., and Mandzhavidze, N. Z.: 1990, Solar Phys. 125, 91.

Hua, X.-M. and Lingenfelter, R. E.: 1987, Solar Phys. 107, 351.

Kocharov, G. E., Kovaltsov, G. A., and Kocharov, L. G.: 1983, Proc. 18th Int. Cosmic Ray Conf., Bangalore, 4, 105.

Kocharov, L. G. and Kovaltsov, G. A.: 1990, Solar Phys. 125, 67.

Kocharov, L. G., Kovaltsov, G. A., Gueglenko, V. G., Kartavyh, Yu. Yu., Kocharov, G. E., and Usoskin, I. G.: 1991, Nuclear Astrophysics, Phys.-Tech. Inst. Publ., St.-Petersburg, Russia, p. 5.

Kocharov, L. G., Lee, J. W., Zirin, H., Kovaltsov, G. A., Usoskin, I. G., Pyle, K. R., Shea, M. A., and Smart, D. F.: 1994, Solar Phys., in press.

Lee, J. W., Gary, D. E., and Zirin, H.: 1994, Solar Phys., in press.

Mandzhavidze. N. Z. and Ramaty. R.: 1992, Astrophys. J. 389, 739.

Mandzhavidze, N. Z. and Ramaty, R.: 1993, Nuclear Physics B (Proc. Suppl.) 33A,B, 141.

Miller, J. and Ramaty, R.: 1989, Astrophys. J. 344, 973.

Muraki, Y., Sakakibara, S., Shibata, S., Murakami, K., Takahashi, K., Pyle, K. R., Sakai, T., and Mitsui, K.: 1993, Proc. Kofu Symposium 1. 
Pelaez, F.: 1993, Thèse de doctorat de l'Université Paul Sabatier, Toulouse, France.

Petrosian, V.: 1985, Astrophys. J. 299, 987.

Pyle, K. R. and Simpson, J. A.: 1991, Proc. 22nd Int. Cosmic Ray Conf., Dublin 3, 53.

Ramaty, R., Mandzhavidze, N., Kozlovsky, B., and Skibo, J. G.: 1993, Adv. Space Res. 13(9), 275.

Ramaty, R., Schwartz, R. A., Enome, S., and Nakajama, H.: 1994, Astrophys. J., to appear.

Talon, G., Trottet, G., Vilmer, N., Barat, C., and Dezalay, J.-P.: 1993, Solar Phys. 147, 137.

Terekhov, O. V., Sunyaev, R. A., Kuznetsov, A. V., Barat, C., Talon, R., Trottet, G., and Vilmer, N.: 1993, Pis'ma v Astron. J. (Soviet Astron. Letters) 19, No. 3, 163.

Vestrand, W. T., Forrest, D. J., Rieger, E.: 1991, Proc. 22nd Int. Cosmic Ray Conf., Dublin 3, 69.

Vestrand, W. T., Forrest, D. J., Chupp, E. L., Rieger, E., and Share, G. H.: 1987, Astrophys, J. 322, 1010.

Vilmer, N., Trottet, G., Barat, C., Dezalay, J. P., Talon, R., Sunyaev, R., Terekhov, O., Kuznetsov, A.: 1994, Lecture Notes in Physics, Proc. 7th Europ. Meeting in Solar Phys., Catania, May 1993, p. 1.

Wang, H., Ewell, M. W., Jr., Zirin, H., and Ai, G.: 1994, Astrophys. J. 424, 436.

Zirin, H.: 1988, Astrophysics of the Sun, Cambridge University Press, Cambridge, p. 358. 